\title{
Desynchronization of Theta Oscillations in Prefrontal Cortex during Self-stimulation of the Medial Forebrain Bundles in Mice
}

\author{
Eunjin Hwang ${ }^{1}$, Heonsoo Lee ${ }^{2}$ and Jee Hyun Choi ${ }^{1,3 *}$ \\ ${ }^{1}$ Center for Neuroscience, Korea Institute of Science and Technology, Seoul 02792, Korea, ${ }^{2}$ Division of Neuroanesthesiology, \\ Department of Anesthesiology, University of Michigan Medical School, Ann Arbor, MI 48109-5048, USA, \\ ${ }^{3}$ Department of Neuroscience, University of Science and Technology, Daejeon 34113, Korea
}

\begin{abstract}
Stimulation of the medial forebrain bundle (MFB) can reinforce intracranial self-stimulation (ICSS) in rodents (i.e., reward-seeking behavior). The MFB stimulation produces a highly reliable behavioral output that enabled a clear distinction of the animal behavioral states between the non-ICSS and ICSS periods. However, the cortical states during these reward-seeking behaviors are not fully characterized in comparison to those during volitional behavior. This study was designed to characterize the cortical rhythms of and coherence between prefrontal cortex and hippocampus during the wheel-turning behavior reinforced by the ICSS in comparison to the wheel-turning without ICSS. We used a wheel for freely moving mice, which was programmed to deliver cathode currents through an electrode in the MFB at each one-quarter turn of the wheel to induce ICSS. The wheel-turning epochs were extracted from the pre-ICSS, ICSS and post-ICSS sessions and the prefrontal EEGs and the hippocampal LFPs in the epochs were analyzed with power and synchronization analyses. During the ICSS, the EEG power decreased at $6 \sim 10 \mathrm{~Hz}$ in the prefrontal cortex, while was not significantly altered in the hippocampus. Furthermore, we found that the phase synchrony between the prefrontal cortex and the hippocampus corresponding to information transmission between the two regions during reward-seeking motion decreased preceding MFB stimulation reinforced by ICSS. Our findings suggest that theta-activity can be reliably dissociated from active behavior if the animal is involved in self-stimulation.
\end{abstract}

Key words: Intracranial self-stimulation (ICSS), Medial forebrain bundles (MFB), Theta rhythms, Prefrontal cortex, Hippocampus, Mice

\section{INTRODUCTION}

Intracranial self-stimulation (ICSS) is a paradigm in which

Received April 12,2018, Revised May 29,2018,

Accepted May 29, 2018

* To whom correspondence should be addressed.

TEL: 82-2-958-6952, FAX: 82-2-958-6737

e-mail: jeechoi@kist.re.kr animals self-administer rewarding brain stimulation through electrodes implanted in reward circuits. The use of ICSS has been practical, particularly in rodents mainly due to their operant ICSS behavior $[1,2]$. However, cortical activities, particularly during reward-seeking behavior, have not been fully analyzed compared to stereotypic patterns during the same but volitional behavior. Typically, a wheel is used for the facilitation of ICSS behavior in mice, and a lever is used in rodents [3]. Although most of the observations were made during learning situations, one of the typical 
brain activities observed during wheel turning is hippocampal activity [4].

In the hippocampus, theta oscillations in the frequency range of $6 \sim 10 \mathrm{~Hz}$ present during active motor behavior, such as locomotion and exploration [5]. It has been known that sensorimotor integration is associated with theta rhythms [6]; in some studies, however, context, rather than motion, has been suggested as being more critical to the generation of theta rhythms. For example, van Lier and his colleagues found that hippocampal theta power was different between voluntary and automatic motions and showed that the non-motor process might involve theta rhythms [7]. In addition, Wyble and his colleagues showed that hippocampal theta power was dependent on the expectation of reward, even in the case of the same behaviors [8].

In regard to reward-seeking behaviors, the prefrontal cortex is known to be critically involved $[9,10]$, and its connectivity to subcortical structures, such as the ventral striatum and midline thalamus, is known to contribute to reward-seeking behaviors [11-13]. While it seems reasonable that the association of a certain behavior to a reward requires learning and memory, in which hippocampus plays an essential role, there is still a lack of understanding of how hippocampal theta oscillations and hippocampalprefrontal interaction are affected by motivational states.

To investigate the involvement of hippocampal and prefrontal theta rhythms in motivational behavior, we induced a rewardseeking wheel-turning behavior in mice by using ICSS of the medial forebrain bundle (MFB), which is a part of the reward system and has been proven to be an effective operant reinforcer. Local field potential (LFP) and electroencephalogram (EEG) data were acquired from the dorsal hippocampus (HPC) and the prefrontal cortex (PFC), respectively, and theta band power during the wheel-turning activities were compared between a motivated condition (with ICSS) and a normal condition (without ICSS). HPC-PFC interactions during the wheel-turning behavior were characterized by a phase synchronization index as well.

\section{MATERIALS AND METHODS}

\section{Animal preparation}

Six male C57BL/6 mice (age $>15$ weeks; body weight $>25$ g) were used in our study. The animals were housed in a colony maintained on a 12 -h light/dark schedule at $22^{\circ} \mathrm{C}$ with $55 \%$ humidity and were allowed ad libitum access to food and water. All surgical and experimental procedures were approved by the Institutional Animal Care and Use Committee of the Korea Institute of Science and Technology (Permit Number: AP-201001048). Animal handling and surgery were performed in accordance with the guidelines of the Korean Animal and Plant Quarantine Agency (Publication No. 12512, partial amendment 2014).

\section{Electrode implantation surgery}

Mice were anesthetized with IP injections of a ketamine-xylazine cocktail (120 and $6 \mathrm{mg} / \mathrm{kg}$, respectively) and were fixed in a stereotaxic apparatus (Model 902, David Kopf Instruments, Tujunga, CA, USA) for the electrode implantation surgery. A screw-type electrode (chrome-plated stainless steel, $1 \mathrm{~mm}$ in diameter, Asia Bolt, Seoul, Korea) was implanted in the right medial prefrontal cortex (PFC), and a needle-type electrode (Teflon-insulated tungsten wire, 0.003/0.0045" in bare/coated diameter, A-M Systems, Sequim, WA, USA) was implanted in the left dorsal hippocampus (HPC). The coordinates for the PFC were AP 2.8, ML +1.5 for three mice and $\mathrm{AP} 1.7, \mathrm{ML}+0.4$ for the other three mice. For the HPC, the coordinates were AP -2.54, ML -3.2, DV - 1.5 for two mice, AP -2.54, ML -2.0, DV - 1.6 for another mouse and AP -1.94, ML - 1.5, DV - 1.4 for the remaining three mice. A stimulating electrode (2-channel twisted electrode with a 0.3 -mm diameter, Plastics One, Roanoke, VA, USA) was implanted in the medial forebrain bundle (MFB) for delivery of the electrical stimulation, and the coordinates were AP -0.34, ML - 1.2, DV -5.0. Mice were given a 7-day recovery period after the surgery and were transferred to self-stimulation training.

\section{Intracranial self-stimulation (ICSS)}

Intracranial self-stimulation (ICSS) was carried out by the wheelturning behavior of mice. Mice were placed in a custom-made chamber (transparent plastic cage, $20 \mathrm{~cm} \times 20 \mathrm{~cm} \times 13 \mathrm{~cm}$ ) with free access to a wheel (See Fig. 1A for a schematic drawing of the experimental set-up) and had at least a 1-hr habituation period before stimulation. A magnetic rotation sensor attached to the wheel determined the moment at which the mice turned the wheel by $1 / 4$ of a rotation and conveyed a trigger signal to a stimulator (Isolated pulse stimulator model 2100, A-M Systems, Carlsborg, WA, USA) to deliver stimulation to the MFB $0.5 \mathrm{~s}$ after the wheel rotation moment. The stimulation was a train of square-wave cathodal pulses at $100 \mathrm{~Hz}$ given continuously until the rotation stopped. Each stimulation current was set individually to a minimum effective current $(50 \sim 150 \mu \mathrm{A})$, which could cause each mouse to sustain wheel-turning behavior. The level of wheel-turning activity was quantified as 1 (rotation) and 0 (no rotation) every second based on the rotation sensor signal. Mice were trained for one hour a day for a couple of weeks to achieve sufficient wheel-turning behavior reinforced by the MFB self-stimulation, until the average wheelturning activity in each stimulation session became larger than 0.4 . It took 15 days for the first three mice, 14 days for two mice, and 

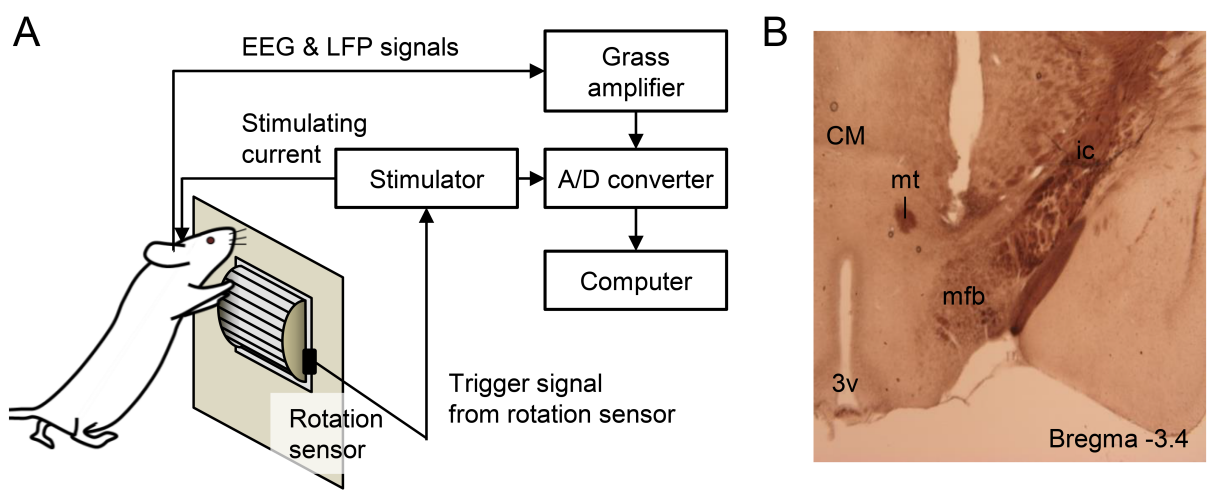

Fig. 1. Experimental setup and histological verification of the stimulation site. (A) Experimental setup for ICSS. A mouse was positioned in a custommade stimulation chamber with free access to a wheel. When the mouse turned the wheel by $1 / 4$ of a rotation, a trigger signal from a rotation sensor was sent to both a pulse stimulator and an analog-to-digital (A/D) converter. The pulse stimulator gave the electrical stimulation to the MFB following a protocol arranged in the stimulator. Prefrontal EEG and hippocampal LFP data were acquired by an analog amplifier, digitalized at $500 \mathrm{~Hz}$ and recorded by a computer. (B) Histological verification of the tip of the stimulating electrode. CM, central medial thalamic nucleus; ic, internal capsule; mt, mammillothalamic tract; $\mathrm{mfb}$, medial forebrain bundle; $3 \mathrm{v}$, third ventricle.

12 days for one mouse to achieve reward-seeking wheel-turning behavior. Electrophysiological signals acquired from the last day when the mice achieved the reward-seeking wheel-turning were analyzed.

\section{Experimental paradigm}

The experiment was composed of three sessions: the pre-ICSS for $5 \mathrm{~min}$, the ICSS for $10 \mathrm{~min}$, and the post-ICSS for $5 \mathrm{~min}$. Electrical stimulation to the MFB was triggered by the wheel-turning behavior of the mice only during the second ICSS session, whereas it was not given during the first and third sessions (stimulation current set to $0 \mu \mathrm{A}$ ). There was no cue or signal informing of the transition between sessions; thus, the mice could notice the change in sessions only by turning the wheel. The experiment was performed twice in succession for each mouse and the behavioral and electrophysiological results from the two were averaged.

\section{Wheel-turning epoch selection}

An epoch was defined as $1 \mathrm{~s}$ before and $1 \mathrm{~s}$ after the onset of wheel-turning activity. The wheel-turning activity which lasted shorter than $2.5 \mathrm{~s}$ was excluded from the analysis. The numbers of epochs detected from all six mice were 148, 484, and 124 during the pre-ICSS, ICSS and post-ICSS sessions, respectively. The onset of wheel-turning activity was estimated based on the recorded stimulation pulse. Since the MFB stimulation was set to be delivered $0.5 \mathrm{~s}$ after the wheel rotation moment, which was detected by the magnetic rotation sensor, the onset of wheel-turning was determined as the moment $0.5 \mathrm{~s}$ before the beginning of the MFB stimulation. In the non-ICSS sessions, the stimulation current was just set to $0 \mu \mathrm{A}$ so that the onset of each wheel rotation could be traced, as in the ICSS session.

\section{Electrophysiological signal acquisition}

Electroencephalogram (EEG) data from the PFC and local field potential (LFP) data from the HPC were amplified by a Grass amplifier (QP511, GRASS Product Group, West Warwick, RI, USA) and were digitized by an analog-digital converter (Digidata 1440A, Molecular Devices, Sunnyvale, CA, USA) at a sampling rate of 500 $\mathrm{Hz}$. The timing of the stimulating pulse train was simultaneously recorded with brain signals.

\section{Power spectrum and phase synchronization}

Signals were analyzed using MATLAB (Mathworks, Natick, MA, USA). Signals were first normalized by the power in the fast frequency band $(90 \sim 100 \mathrm{~Hz})$ to make the voltage levels acquired from different animals with different types of electrodes match, and downsampled to $200 \mathrm{~Hz}$ prior to further analysis. The epochs that had signal amplitudes larger than 5SD of the entire recording sessions (pre-ICSS, ICSS and post-ICSS) were excluded from the analysis.

Power spectrum data were obtained by Welch's method for the period after the onset of wheel-turning activity of each epoch. Spectrograms were computed by wavelet analyses (complex Morlet wavelet with seven cycles as a mother function), where the power was obtained by taking the square of the wavelet coefficient. The theta band $(6 \sim 10 \mathrm{~Hz})$ power before and after the onset of wheel-turning activity and the onset of the MFB stimulation was computed in three time bins and compared among the time bins or among the pre-ICSS, ICSS and post-ICSS sessions.

The phase synchronization index (PSI) between the PFC and the 
HPC was calculated as PSI $=\frac{1}{N}\left|\sum_{\mathrm{k}=1}^{\mathrm{N}} \mathrm{e}^{-\mathrm{i} \Delta \phi_{\mathrm{k}}(\mathrm{t})}\right|$, where $\Delta \phi_{\mathrm{k}}(\mathrm{t})$ is a phase difference between signals recorded in the PFC and the $\mathrm{HPF}$ at time $\mathrm{t}$ in $\mathrm{k}$-th wheel-turning epoch, and $\mathrm{N}$ is the number of the epochs [14]. Phases were computed by Hilbert transformation of the theta bandpass-filtered signals.

\section{RESULTS}

\section{Increased wheel-turning activity during ICSS}

Mice were placed in a custom-made stimulation chamber with free access to a wheel (Fig. 1A) and the stimulation to MFB (See Fig. 1B for the histological verification of the tip position) was triggered by the wheel-turning behavior of mice during the ICSS sessions. As seen in Fig. 2A, the mice showed a persistent increase
A

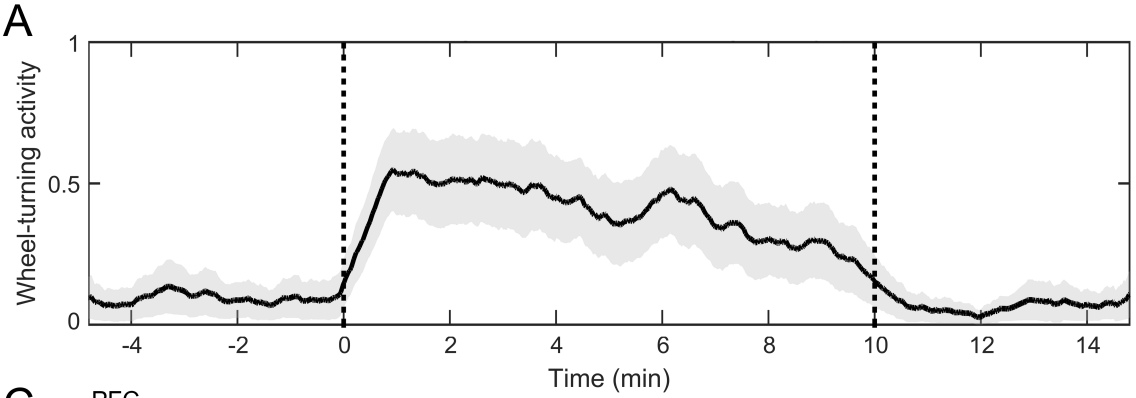

C

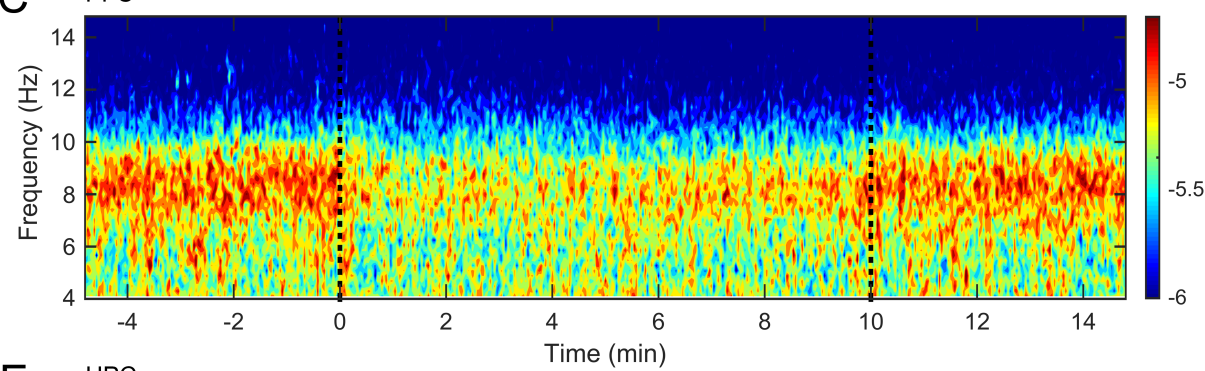

E

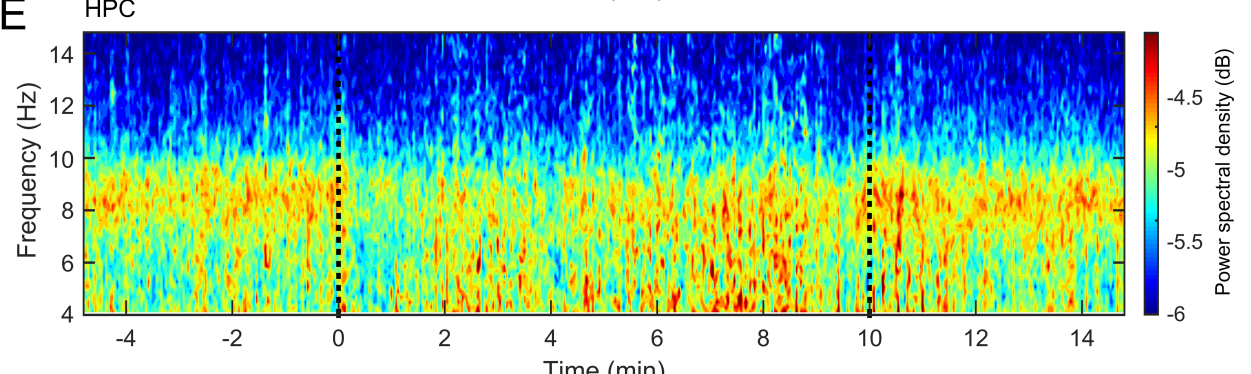

B
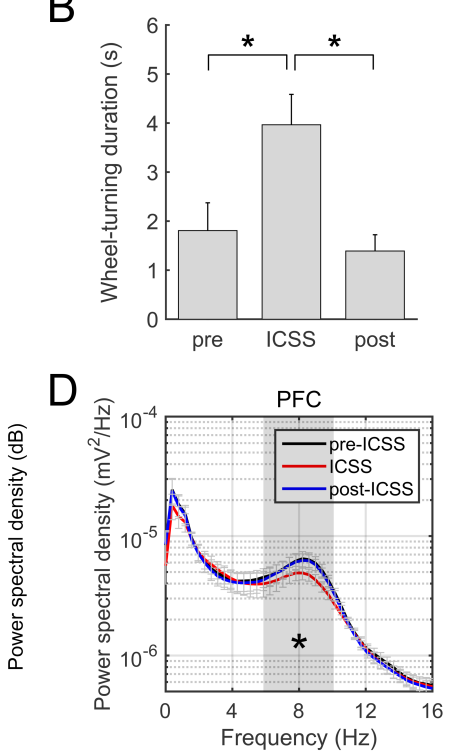

$\mathrm{F}$

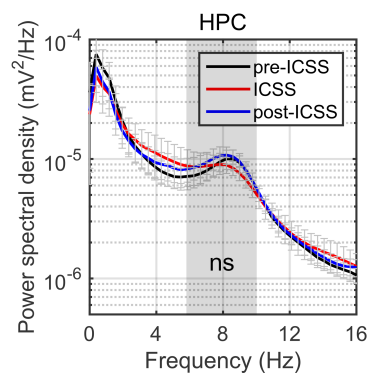

G

pre-ICSS

ICSS

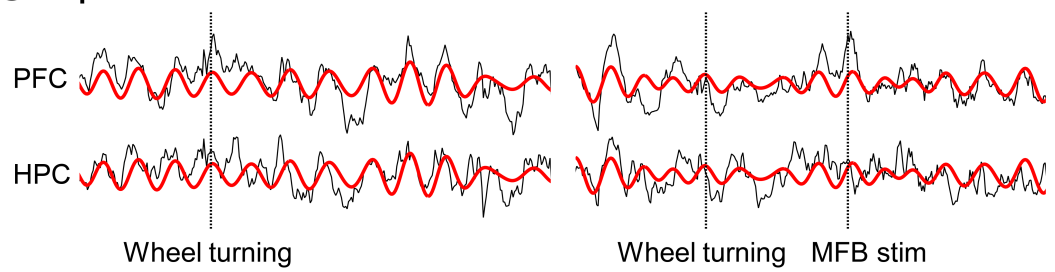

Wost-ICSS - Raw

Fig. 2. Theta power modulation by ICSS. (A) Average trace of the wheel-turning activity of six mice. Dotted vertical lines indicate the onset (time $=0$ ) and the cessation (time $=10$ ) of the MFB stimulation. Gray shades for SEM. (B) Mean duration of the wheel-turning activity. ${ }^{*}$ for $\mathrm{p}<0.05$, repeated measures ANOVA and post hoc comparison with the Tukey-Kramer method. Error bars for SEM. (C, E) Average spectrograms of the PFC EEG and HPC LFP data. (D, F) Average power spectral density during the pre-ICSS, the ICSS and the post-ICSS sessions in the PFC and the HPC, respectively. Gray shades indicate the frequency range of the theta rhythm $(6 \sim 10 \mathrm{~Hz})$, where a significant difference was found in the PFC between the pre-ICSS and ICSS sessions. ${ }^{*}$ for $\mathrm{p}<0.05$, Wilcoxon signed rank test. Error bars for SEM. (G) Representative traces of the raw signals and corresponding theta-filtered signals for the PFC EEG and the HPC LFP data. Scale of the EEG and LFP data were matched by normalization. 
in wheel-turning activity during the ICSS sessions (from time 0 to $10 \mathrm{~min}$ ), compared to the pre- and the post-ICSS sessions. The mean durations of wheel turning were $1.81 \pm 0.57 \mathrm{~s}, 3.96 \pm 0.62 \mathrm{~s}$, and $1.39 \pm 0.33 \mathrm{~s}$ during the pre-ICSS, ICSS, and post-ICSS sessions, respectively (Fig. 2B). There were statistically significant differences in the durations between the pre-ICSS and ICSS sessions and between the post-ICSS and ICSS sessions $(p<0.05$, repeated measures ANOVA and post hoc comparison with the TukeyKramer method). There was no significant difference between the pre-ICSS and post-ICSS sessions $(\mathrm{p}=1.00)$. The supplementary videos show a mouse turning the wheel in a compulsive manner to receive the stimulation during the ICSS session, while it does not in the pre-ICSS session, showing a relaxed and explorative approach.

\section{Reduced theta power during ICSS}

In Figs. 2C and 2E, the spectrograms show a visible change in theta power both in the PFC and the HPC during ICSS. The power spectrum revealed that the PFC theta power during the ICSS session was reduced compared to that during the pre-ICSS session (Fig. 2D; $\mathrm{p}=0.03$, Wilcoxon signed rank test). The HPC theta power was not significantly reduced but showed a trend to decrease (Fig. 2F; $\mathrm{p}=0.06$, Wilcoxon signed rank test). To investigate any effects of the wheel-turning activity to the theta power modulation, we extracted the wheel-turning epochs from 1-s before to 1-s after the onset of wheel-turning activity. Representative raw and thetafiltered signals before and after the onset of wheel-turning activity during the pre-ICSS, ICSS and post-ICSS sessions are presented in Fig. 2G.

When we closely examine the periods before and after the onset of wheel-turning activity (Fig. 3A for average spectrogram and $3 \mathrm{~B}$
A
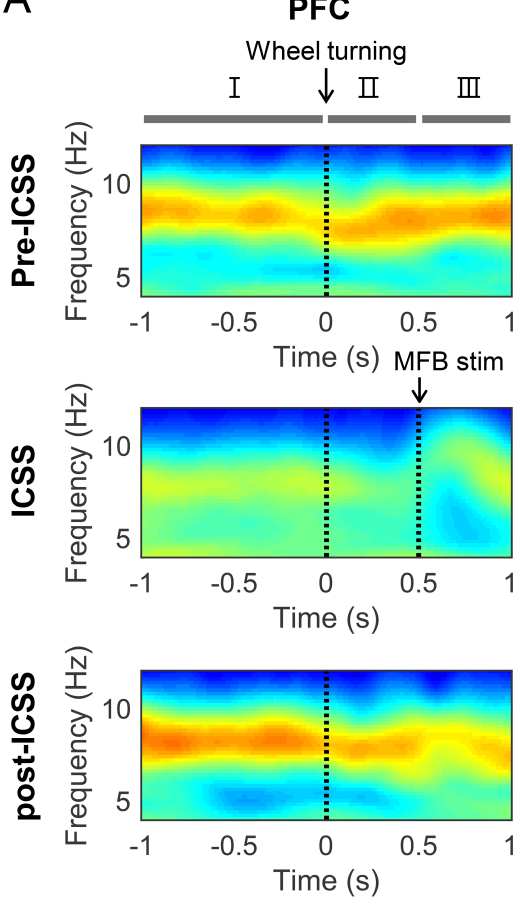

B

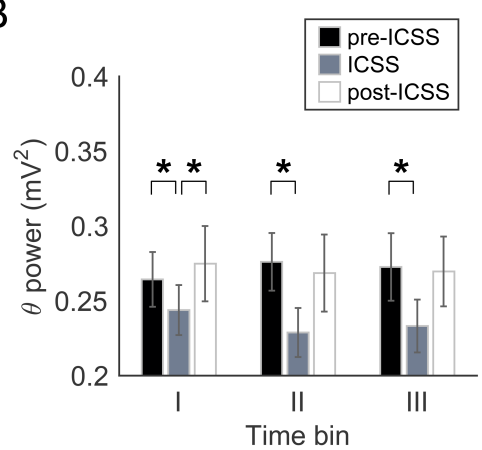

HPC
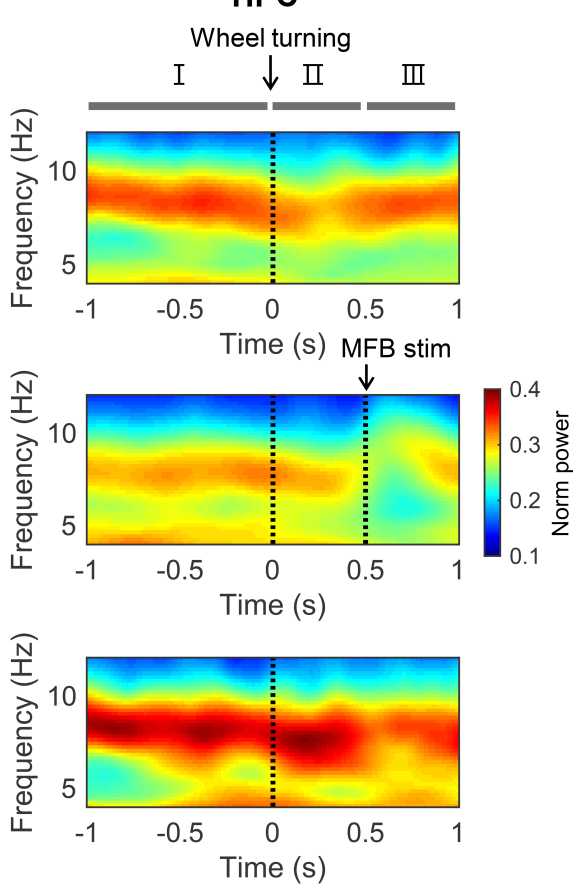

*

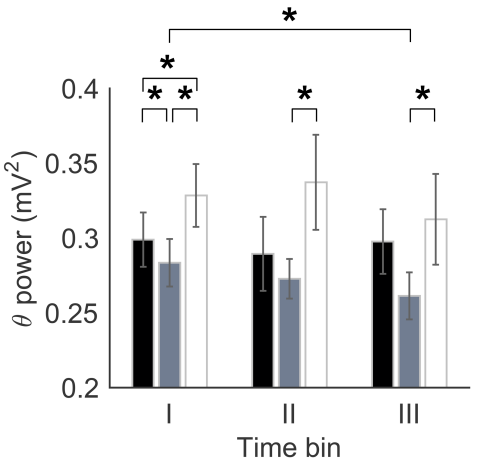

Fig. 3. Theta power modulation during the wheel-turning activity. (A) Average wavelet spectrogram around the onset of wheel-turning activity for the PFC (left) and the HPC (right). Dotted vertical lines at time 0 indicate the onset. For the ICSS session in the second row, the onset of the MFB stimulation is also marked with dotted vertical lines. (B) Theta power in the time bins I III. ${ }^{*}$ for $\mathrm{p}<0.05$, Wilcoxon signed rank test. Error bars for SEM. 
for summarizing bar graphs), theta power was not significantly modulated temporally except the period when MFB stimulation was given. The theta power during the stimulus-free wheelturning (time bin II) activity was not significantly different from baseline (time bin I) for both the PFC and the HPC during the ICSS session ( $\mathrm{p}=0.06$ for both, Wilcoxon signed rank test) as well as the pre- and the post-ICSS sessions. The HPC theta power was reduced after the stimulation onset (time bin III) in the ICSS session, while the PFC theta power in time bin III was not significantly different from the baseline level in the ICSS. On the other hand, the basal theta power (time bin I) was significantly lower $(\mathrm{p}<0.05$, Wilcoxon signed rank test) in the ICSS session compared to the pre-ICSS session for both the PFC and the HPC. The basal PFC theta power was comparable for both the pre- and the postICSS sessions, while the basal HPC theta power was enhanced for the post-ICSS session ( $\mathrm{p}=0.03$, Wilcoxon signed rank test). The decrease in basal theta power might indicate an association of the theta power with the expectation of reward, regardless of the effect of MFB stimulation or the wheel-turning behavior on the theta power.

\section{Decreased phase synchrony between the PFC and the HPC during ICSS}

The phase synchrony between the PFC and the HPC was comparable among the sessions before the onset of wheel-turning activity but was deviated in the interval from 0.4 to $0.7 \mathrm{~s}$ after the onset (Fig. 4A). During the interval $0.4 \sim 0.5 \mathrm{~s}$ which is before the stimulation onset (Fig. 4B), the PSI was significantly different between the pre-ICSS and the ICSS sessions ( $\mathrm{p}=0.03$, repeated measures ANOVA and post hoc comparison with the Tukey-Kramer method). The PSI difference was also found to be significant between the pre-ICSS and the ICSS sessions during the interval $0.5 \sim 0.7 \mathrm{~s}$ (Fig. $4 \mathrm{C} ; \mathrm{p}=0.03$, repeated measures ANOVA and post hoc comparison with the Tukey-Kramer method). Compared to the baseline PSI obtained from the interval between -1.0 and $0 \mathrm{~s}$, the PSI was not significantly different during both of the intervals for all the sessions ( $p>0.05$, Wilcoxon signed rank test).

\section{DISCUSSION}

We have shown that the theta rhythms behind the seemingly equivalent behavior of wheel-turning can be different depending on the behavior state during ICSS paradigm in mice. By using the ICSS paradigm, which starts to deliver the stimulation current $0.5 \mathrm{~s}$ after the onset of wheel-turning activity, we could compare the electrophysiological features of an equivalent and simple stimulus-free behavior between a reward-seeking condition and in a volitionally behaving condition. When the mice turn the wheel without any reward, the theta rhythms were consistently present before and after the wheel-turning activity in both the PFC and the HPC. On the other hand, theta power during the ICSS session showed an eventrelated desynchronization in PFC.

The reduction in theta rhythms we observed in this study might indicate that the wheel-turning behavior became automatic or
A

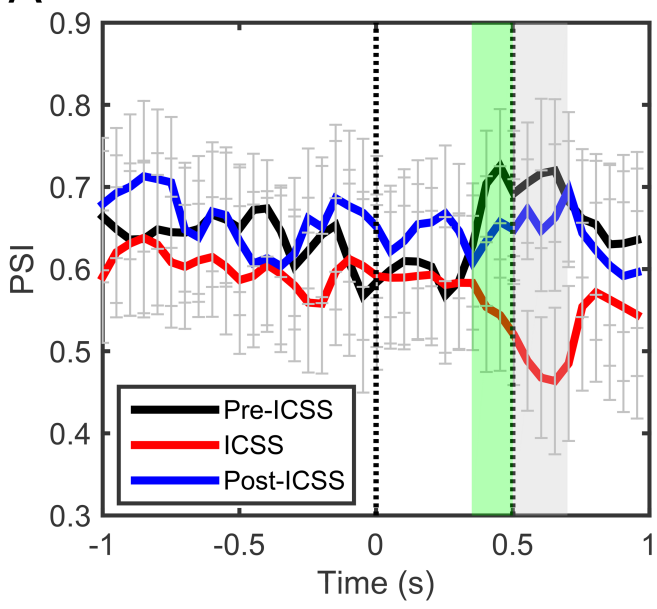

B

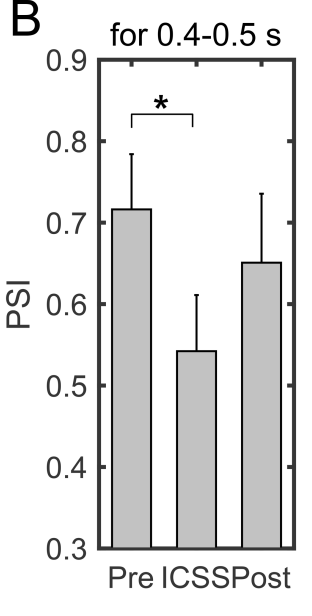

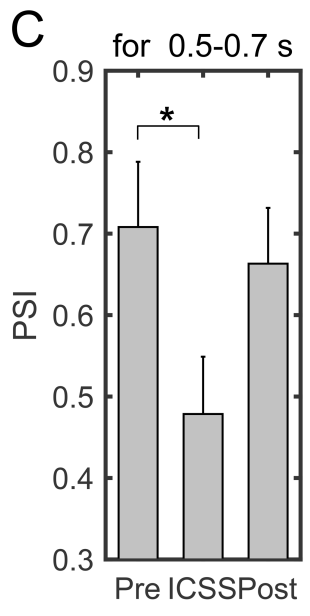

Fig. 4. Modulation of the theta synchronization between the PFC and the HPC. (A) Average PSI between the PFC and the HPC calculated at each timepoint of the wheel-turning epoch. Error bars for SEM. Dotted vertical lines at time 0 and time 0.5 indicate the onset of wheel-turning activity and the onset of MFB stimulation, respectively. Green and gray shades indicate the intervals in which the PSIs in the ICSS session deviated from the pre-ICSS sessions before and after the stimulation onset, respectively. (B) Mean PSIs in the interval from 0.4 to 0.5 s. Error bars for SEM. (C) Mean PSIs in the interval 0.5 to $0.7 \mathrm{~s}$. ${ }^{\star}$ for $\mathrm{p}<0.05$, repeated measures ANOVA and post hoc comparison with the Tukey-Kramer method. 
consummatory by ICSS. Since the theta rhythms are known to appear with active motor behavior, at a glance, it seems contrary to the typical pattern that the theta rhythms decreased during the ICSS session where the mice turned the wheel very actively. However, researchers have also found that the regular theta rhythms are associated with the control of voluntary movement [15], and the brain oscillations become irregular when the animals perform automatic behaviors such as grooming, chewing and licking [5, 16]. Compared to consummatory behaviors, which pursue the satisfaction of innate drive, the sequence of movements that compose voluntary behaviors is not fixed, thus, involving a more complex neural circuit [5]. It is also known that the two types of hippocampal oscillations associated with different types of behaviors have distinct cellular correlates; granule cells in the dentate gyrus discharge the most during exploratory and voluntary behaviors, while pyramidal neurons in the CA fields are maximally active during consummatory behaviors [17]. ICSS might simplify the circuit involved in wheel-turning activity (which is exploratory in nature per se) and the change in neural activities would be reflected in EEG and LFP.

The change in the phase synchrony between the PFC and the HPC during the ICSS session also supports a possible change in the neural circuit related to the wheel-turning behavior. The PFC and the dorsal HPC communicate to each other either directly or indirectly through nucleus reuniens during memory processes [18], and it has been reported that the level of theta synchronization is associated with encoding [19] and retrieval [20] of memories. Decreased synchronization reflects reduced information transmission between the PFC and the HPC, possibly indicating a change in the memory retrieval process during automatic behavior.

Our study was limited to investigating only a part of reward circuitry but was still able to show that the motion-related theta rhythms in the PFC and the HPC could be modulated with the motivational states induced by ICSS. Extended research incorporating other regions, such as the ventral tegmental area and the nucleus accumbens, might broaden the circuit-level understanding of reward-seeking behaviors.

\section{ACKNOWLEDGEMENTS}

This research was supported by the National Research Council of Science and Technology of Korea for the project Development of Solution for Diagnosis, Treatment and Care system of Dementia (No. CRC-15-04-KIST) and the National Research Foundation of Korea (NRF) grant funded by the Korea government (2017R1A2B3012659).

\section{REFERENCES}

1. McMurray MS, Conway SM, Roitman JD (2017) Brain stimulation reward supports more consistent and accurate rodent decision-making than food reward. eNeuro 4:ENEURO.0015-17.2017.

2. Donahue RJ, Muschamp JW, Russo SJ, Nestler EJ, Carlezon WA Jr (2014) Effects of striatal $\Delta$ FosB overexpression and ketamine on social defeat stress-induced anhedonia in mice. Biol Psychiatry 76:550-558.

3. Carlezon WA Jr, Chartoff EH (2007) Intracranial self-stimulation (ICSS) in rodents to study the neurobiology of motivation. Nat Protoc 2:2987-2995.

4. O’keefe J, Nadel L (1978) The hippocampus as cognitive map. Oxford, UK, Clarendon Press.

5. Vanderwolf CH (1969) Hippocampal electrical activity and voluntary movement in the rat. Electroencephalogr Clin Neurophysiol 26:407-418.

6. Bland BH, Oddie SD (2001) Theta band oscillation and synchrony in the hippocampal formation and associated structures: the case for its role in sensorimotor integration. Behav Brain Res 127:119-136.

7. van Lier H, Coenen AM, Drinkenburg WH (2003) Behavioral transitions modulate hippocampal electroencephalogram correlates of open field behavior in the rat: support for a sensorimotor function of hippocampal rhythmical synchronous activity. J Neurosci 23:2459-2465.

8. Wyble BP, Hyman JM, Rossi CA, Hasselmo ME (2004) Analysis of theta power in hippocampal EEG during bar pressing and running behavior in rats during distinct behavioral contexts. Hippocampus 14:662-674.

9. Matsumoto K, Tanaka K (2004) The role of the medial prefrontal cortex in achieving goals. Curr Opin Neurobiol 14:178-185.

10. Otis JM, Namboodiri VM, Matan AM, Voets ES, Mohorn EP, Kosyk O, McHenry JA, Robinson JE, Resendez SL, Rossi MA, Stuber GD (2017) Prefrontal cortex output circuits guide reward seeking through divergent cue encoding. Nature 543:103-107.

11. Cardinal RN, Parkinson JA, Hall J, Everitt BJ (2002) Emotion and motivation: the role of the amygdala, ventral striatum, and prefrontal cortex. Neurosci Biobehav Rev 26:321-352.

12. Ma YY, Lee BR, Wang X, Guo C, Liu L, Cui R, Lan Y, BalcitaPedicino JJ, Wolf ME, Sesack SR, Shaham Y, Schlüter OM, Huang YH, Dong Y (2014) Bidirectional modulation of incubation of cocaine craving by silent synapse-based remodeling of prefrontal cortex to accumbens projections. Neuron 
83:1453-1467.

13. McFarland K, Kalivas PW (2001) The circuitry mediating cocaine-induced reinstatement of drug-seeking behavior. J Neurosci 21:8655-8663.

14. Lachaux JP, Rodriguez E, Martinerie J, Varela FJ (1999) Measuring phase synchrony in brain signals. Hum Brain Mapp 8:194-208

15. Whishaw IQ, Vanderwolf CH (1973) Hippocampal EEG and behavior: changes in amplitude and frequency of RSA (theta rhythm) associated with spontaneous and learned movement patterns in rats and cats. Behav Biol 8:461-484.

16. Sainsbury RS (1970) Hippocampal activity during natural behavior in the guinea pig. Physiol Behav 5:317-324.

17. Buzsáki G (1989) Two-stage model of memory trace formation: a role for "noisy" brain states. Neuroscience 31:551-570.

18. Sigurdsson T, Duvarci S (2016) Hippocampal-prefrontal interactions in cognition, behavior and psychiatric disease. Front Syst Neurosci 9:190.

19. Sigurdsson T, Stark KL, Karayiorgou M, Gogos JA, Gordon JA (2010) Impaired hippocampal-prefrontal synchrony in a genetic mouse model of schizophrenia. Nature 464:763-767.

20. Jones MW, Wilson MA (2005) Theta rhythms coordinate hippocampal-prefrontal interactions in a spatial memory task. PLoS Biol 3:e402. 